\title{
Infectious mononucleosis and hepatic function
}

\author{
LI ZHANG $^{1^{*}}$, PINGPING ZHOU ${ }^{2 *}$, ZHAOWEI MENG ${ }^{2}$, CHONGJIE PANG $^{1}$, \\ $\mathrm{LU} \mathrm{GONG}^{1}$, QING ZHANG ${ }^{3}$, QIYU JIA ${ }^{3}$ and KUN SONG ${ }^{3}$ \\ Departments of ${ }^{1}$ Infectious Diseases, ${ }^{2}$ Nuclear Medicine and ${ }^{3}$ Health Management, \\ Tianjin Medical University General Hospital, Tianjin 300052, P.R. China
}

Received August 12, 2017; Accepted November 9, 2017

DOI: $10.3892 / \mathrm{etm} .2018 .5736$

\begin{abstract}
Abnormal hepatic function is common in infectious mononucleosis (IM). However, it remains unknown why increased transferase levels are more common than bilirubin abnormalities in IM. The current study aimed to investigate these associations in the Chinese population. A total of 95 patients with IM ( 47 males and 48 females) were enrolled in the current study, as well as 95 healthy controls. Patients were sorted by sex. A receiver operating characteristic (ROC) curve was used to determine cut-off values for IM diagnosis and prediction. Crude and adjusted odds ratios (OR) for IM were analyzed using binary logistic regression. It was determined that alanine aminotransferase (ALT), aspartate aminotransferase (AST) and $\gamma$-glutamyl transferase (GGT) levels were significantly higher in patients with IM compared with controls; however, total bilirubin (TB) levels were significantly lower in patients with IM. ROCs demonstrated that, if ALT, AST and GGT concentrations were higher than, or if TB was lower than, cut-off values, they were predictive of IM. Binary logistic regression identified that the risk of IM in patients exhibiting high levels of transferases was significantly increased, particularly in males. Crude ORs in ALT quartile 4 were 21.667 and 10.111 for males and females, respectively and adjusted ORs were 38.054 and 9.882, respectively. A significant IM risk of IM was evident in patients with low bilirubin levels and
\end{abstract}

Correspondence to: Dr Zhaowei Meng, Department of Nuclear Medicine, Tianjin Medical University General Hospital, 154 Anshan Road, Tianjin 300052, P.R. China

E-mail: jamesmencius@163.com

*Contributed equally

Abbreviations: IM, infectious mononucleosis; EBV, Epstein-Barr virus; ALT, alanine aminotransferase; AST, aspartate aminotransferase; GGT, $\gamma$-glutamyl transferase; BH, body height; $\mathrm{BW}$, body weight; BMI, body mass index; WBC, white blood cell; $\mathrm{RBC}$, red blood cell; $\mathrm{Hg}$, hemoglobin; PLT, platelet; TB, total bilirubin; BUN, blood urea nitrogen; $\mathrm{Cr}$, creatinine; ROC, receiver operating characteristic; OR, odds ratio; CI, confidence interval

Key words: infectious mononucleosis, Epstein-Barr virus, bilirubin, transferase females appeared to be particularly susceptible. For example, crude ORs in quartile 1 were 8.229 and 8.257 for males and females, respectively and adjusted ORs were 8.883 and 10.048 , respectively. Therefore, the current study identified a positive association between transferase levels and IM and a negative association between TB and IM. Therefore, the results of the current study indicate that high transferases are suggestive of IM, particularly in males, whereas low TB is suggestive for IM, particularly in females.

\section{Introduction}

Infectious mononucleosis (IM) is a common disease associated with Epstein-Barr virus (EBV) infection and $\sim 5$ cases occur per 1,000 people every year $(1,2)$ The results of a survey conducted in Beijing indicated that the incidence of IM peaks among preschool children and young people aged 15-24 years old (3). Diagnosis of IM is generally based on identifying the triad of clinical manifestations of fever, pharyngitis and lymphadenopathy in patients, as well as a positive result following a serological test for EBV $(1,2)$. However, patients with IM also exhibit other symptoms, including headache, fatigue, exanthema, hepatomegaly and splenomegaly (4). The majority of patients with IM recover without complications and return to their normal activities $\sim 2$ months following disease onset $(1,2)$. However, severe complications, including upper-airway obstruction, hemolytic anemia, thrombocytopenia, hepatitis, myocarditis, splenic rupture, neurological and hematological complications may occur in certain cases $(1,2)$. The treatment for IM is generally supportive and only alleviates symptoms, as EBV cannot be cured; these treatments include glucose and sodium chloride intravenous infusion, reduced glutathione intravenous infusion, pantoprazole sodium injection, and oral loxoprofen and bicyclol tablets intake $(1,2)$. Abnormality of hepatic function is commonly encountered in IM; however, it has been demonstrated for many years that increases in alanine aminotransferase (ALT), aspartate aminotransferase (AST) and $\gamma$-glutamyl transferase (GGT) occur more frequently in IM than bilirubin abnormalities (1-12). However, the underlying reasons for these elevations remain unknown. The results of previous studies demonstrated that $>80 \%$ of patients with IM presented with abnormal transferase levels, particularly ALT, but jaundice was uncommon $(1,2,13)$. In addition, Hu et al (14) indicated that the presence of atypical lymphocytes and transaminase may be regarded as a diagnostic marker of IM. 
The aim of the current study was to analyze the associations between hepatic function parameters and IM in a comprehensive manner. The aim was to identify whether abnormal hepatic function could be indicative of IM and whether there were any significant differences between hepatic function parameters between males and females.

\section{Patients and methods}

Patients. Between December 2014 and December 2015, 95 patients (mean age, 39.36 years; 47 males and 48 females) with a confirmed diagnosis of IM were enrolled in the Department of Infectious Diseases, Tianjin Medical University General Hospital (Tianjin, China). Patients with pre-existing liver diseases, including viral hepatitis, fatty liver disease, alcoholic liver disease and medication-induced liver damage, were excluded from the current study. During the same period, a normal control cohort consisting of 95 healthy participants (mean age, 39.45 years; 47 males and 48 females) was recruited from the Health Management Department, Tianjin Medical General University. None of the healthy participants had been diagnosed with any diseases and had attended Tianjin Medical General University for an annual health checkup. The ethical, methodological and protocol aspects of the current investigation were approved by the Institutional Review Board of Tianjin Medical University General Hospital and written informed consent was provided by all participants.

Parameter measurements. Following admittance of patients with IM to the Department of Infectious Diseases, blood tests and anthropometric measurements were conducted. For participants in the control group, blood tests and anthropometric measurements were completed during their visit to the hospital. Physical examinations included measurements of body height $(\mathrm{BH})$ and body weight $(\mathrm{BW})$. Subsequently, body mass index (BMI; $\left.\mathrm{kg} / \mathrm{m}^{2}\right)$ was calculated using the following formula: $\mathrm{BW} /\left(\mathrm{BH}^{2}\right)$. Fasting blood tests were performed following venipuncture and the following serological parameters were measured: White blood cell (WBC), red blood cell (RBC), hemoglobin (Hg) and platelet (PLT) levels. A total of $3 \mathrm{ml}$ of whole blood samples were collected and analyzed using a hemocytometer analyzer (Sysmex Corporation, Kobe, Japan). ALT, AST, GGT, total bilirubin (TB), blood urea nitrogen $(\mathrm{BUN})$ and creatinine $(\mathrm{Cr})$ levels were measured using the Hitachi Model 7170 analyzer (Hitachi Ltd., Tokyo, Japan). Levels of immunoglobulin (Ig)M and IgG antibodies against specific EBV antigens were measured by ELISA using three commercial kits (viral capsid antigen antibody IgM, cat. no. E170606AK; viral capsid antigen antibody IgG, cat. no. E170706BO; viral nuclear antigen antibody IgG, cat. no. E170706BR) supplied by (Euroimmun; Medizinische Labordiagnostika AG, Lübeck, Germany). Ultrasonography on neck lymph nodes and abdominal organs was performed the using PHILIPS HD11 XE (Philips, Amsterdam, The Netherlands).

Diagnostic criteria. IM was generally diagnosed based on clinical presentations of a fever, pharyngitis, lymphadenopathy, the presence of atypical lymphocytes on a peripheral blood smear and a positive heterophile antibody test. The majority of the included patients referred to Tianjin Medical University General Hospital (Tianjin, China) were diagnosed with IM in local hospitals prior to recruitment. In order to establish definite IM diagnosis, serological testing for the identification of antibodies against specific EBV antigens was required by the aforementioned ELISA kits $(1,2)$.

Statistical analysis. All data for males and females were analyzed separately and presented as either the mean \pm standard deviation or the median (lower quartile, upper quartile). Differences of parameters between groups were measured using an independent samples $\mathrm{T}$ test or a Mann-Whitney test. Pearson's bivariate correlation analysis was performed between hepatic function indices and other variables. Receiver operating characteristic (ROC) curves were constructed and diagnostic efficacies were determined using optimal sensitivity and specificity values calculated from hepatic function indices. To compare ROC curves, a previously described protocol was used (15). By stratifying data with quartiles of hepatic function indices, the odds ratio (OR) for IM with a $95 \%$ confidence interval (CI) was calculated using binary logistic regression models. OR adjustments were completed by using age and BMI as covariates in a logistic regression model. The Statistical Package for Social Sciences (SPSS v17.0, Chicago, IL, USA) was used for statistical analysis and $\mathrm{P}<0.05$ was determined to indicate a significant difference.

\section{Results}

Patient clinicopathological characteristics. Parameters were compared in males and females separately (Tables I and II). In males, WBC, ALT, AST and GGT were significantly higher in patients with IM than in healthy controls (Table I). However, RBC, Hg and TB were significantly lower in males with IM compared with controls. In females, ALT, AST and GGT were significantly higher in females with IM than in controls (Table II). However, RBC, Hg, TB, BUN and $\mathrm{Cr}$ were significantly lower in females with IM compared with controls.

Correlations between key variables. Correlation coefficients between hepatic function indices and other parameters were calculated in patients with IM (Table III). In males, ALT was positively correlated with WBC, AST and GGT, whereas in females, ALT was positively correlated with AST and GGT. AST was positively correlated with WBC, ALT and GGT in males and positively correlated with ALT and GGT in females. GGT was positively correlated with WBC, ALT and AST in males and positively correlated with age, BMI, WBC, ALT and AST in females. In males, TB only exhibited a positive correlation with $\mathrm{Hg}$; however in females, $\mathrm{TB}$ was positively correlated with $\mathrm{RBC}, \mathrm{Hg}$ and $\mathrm{Cr}$.

Diagnostic or predicative values of hepatic function indices for IM. From ROC analyses, all hepatic function indices exhibited significant diagnostic or predicative values for IM (Fig. 1 and Table IV). If ALT, AST and GGT concentrations were higher than the cut-off values of 27.5, 21.5 and 29.5 U/1 
Table I. Parameter characteristics in males.

\begin{tabular}{|c|c|c|c|}
\hline Parameters & Patients with IM & Controls & $\mathrm{T}$ value/ $\mathrm{Z}$ value \\
\hline Case no. & 47 & 47 & \\
\hline Age (years) & $37.40 \pm 16.83$ & $37.68 \pm 16.83$ & -0.080 \\
\hline BMI $\left(\mathrm{kg} / \mathrm{m}^{2}\right)$ & $23.58 \pm 3.02$ & $24.74 \pm 3.50$ & -1.725 \\
\hline WBC $\left(\times 10^{9} / 1\right)$ & $7.17 \pm 3.81$ & $5.93 \pm 1.49$ & $2.085^{\mathrm{a}}$ \\
\hline $\mathrm{RBC}\left(\times 10^{12} / \mathrm{l}\right)$ & $4.46 \pm 0.51$ & $5.19 \pm 0.38$ & $-7.870^{\mathrm{b}}$ \\
\hline $\mathrm{Hg}(\mathrm{g} / \mathrm{l})$ & $133.19 \pm 14.59$ & $155.15 \pm 10.21$ & $-8.453^{b}$ \\
\hline $\operatorname{PLT}\left(\times 10^{9} / 1\right)$ & $208.47 \pm 80.43$ & $214.81 \pm 46.92$ & -0.467 \\
\hline ALT (U/l) & $50.00(22.00,107.00)$ & $19.00(14.00,27.00)$ & $4.411^{\mathrm{b}}$ \\
\hline AST (U/l) & $31.00(19.00,63.00)$ & $18.00(14.00,22.00)$ & $4.216^{\mathrm{a}}$ \\
\hline GGT (U/1) & $57.00(25.00,123.00)$ & $20.00(16.00,29.00)$ & $5.164^{\mathrm{b}}$ \\
\hline $\mathrm{TB}(\mu \mathrm{mol} / \mathrm{l})$ & $9.50(7.10,11.90)$ & $11.70(9.90,17.90)$ & $2.934^{\mathrm{b}}$ \\
\hline BUN (mmol/l) & $4.10 \pm 1.74$ & $4.56 \pm 1.22$ & -1.496 \\
\hline $\mathrm{Cr}(\mu \mathrm{mol} / \mathrm{l})$ & $76.30 \pm 28.92$ & $83.34 \pm 12.75$ & -1.528 \\
\hline
\end{tabular}

Table II. Parameter characteristics in females.

\begin{tabular}{lcc}
\hline Parameters & Patients with IM & Controls \\
\hline Case no. & 48 & 48 \\
Age $($ years $)$ & $41.27 \pm 17.54$ & $41.19 \pm 17.35$ \\
BMI $\left(\mathrm{kg} / \mathrm{m}^{2}\right)$ & $23.52 \pm 3.81$ & $22.60 \pm 2.94$ \\
WBC $\left(\mathrm{x} 10^{9} / \mathrm{l}\right)$ & $6.29 \pm 3.62$ & $5.17 \pm 1.54$ \\
RBC $\left(\mathrm{x} 10^{12} / \mathrm{l}\right)$ & $3.93 \pm 0.43$ & $4.44 \pm 0.27$ \\
Hg $(\mathrm{g} / \mathrm{l})$ & $113.54 \pm 13.34$ & $130.73 \pm 8.49$ \\
PLT $\left(\mathrm{x} 10^{9} / \mathrm{l}\right)$ & $235.65 \pm 90.10$ & $226.15 \pm 50.15$ \\
ALT $(\mathrm{U} / \mathrm{l})$ & $20.00(12.00,68.50)$ & $13.00(9.25,15.75)$ \\
AST $(\mathrm{U} / \mathrm{l})$ & $21.50(16.00,49.00)$ & $15.50(13.00,18.75)$ \\
GGT $(\mathrm{U} / \mathrm{l})$ & $25.50(13.00,49.50)$ & $9.70(7.65,12.28)$ \\
TB $(\mu$ mol/l) & $6.10(4.90,7.55)$ & $4.15 \pm 1.15$ \\
BUN (mmol/l) & $2.97 \pm 1.05$ & $60.77 \pm 9.59$ \\
Cr $(\mu$ mol/l) & $52.13 \pm 9.46$ & -10.975 \\
\hline Data presented as mean \pm standard deviation or median $\left(\right.$ lower quartile, upper quartile). ${ }^{\mathrm{a}} \mathrm{P}<0.01$ (analyzed by independent sample's t test or \\
Mann-Whitney test). IM, infectious mononucleosis; BMI, body mass index; WBC, white blood cell; RBC, red blood cell; Hg, hemoglobin; \\
PLT, platelet; ALT, alanine aminotransferase; AST, aspartate aminotransferase; GGT, $\gamma$-glutamyl transferase; TB, total bilirubin; BUN, blood \\
urea nitrogen; Cr, creatinine.
\end{tabular}

in males, diagnostic accuracies of IM were 74.468, 71.277 and $75.532 \%$, respectively. In females, the cut-off values for ALT, AST and GGT were 15.5, 17.5 and $18.0 \mathrm{U} / 1$ and diagnostic accuracies of IM were $67.708,66.667$ and $72.917 \%$, respectively. If TB concentrations were lower than the cut-off values of $10.70 \mu \mathrm{mol} / 1$ in males and $7.45 \mu \mathrm{mol} / 1$ in females, diagnostic accuracies were 64.89 and $78.125 \%$, respectively. Comparison of ROC curves in males and females did not identify any evident differences.
Risks of IM in different hepatic function status. Binary logistic regression models were utilized to calculate the risks of IM in the males and females (Table V). For transferases, crude OR calculations were performed using the lowest quartiles as references. High transferase levels significantly increased IM risk, particularly in males. Following adjustments using age and BMI as covariates, males with high levels of transferases exhibited a much greater susceptibility to IM. Crude ORs in ALT quartile 4 were 21.667 and 10.111 for males and females, respectively; however 
Table III. Pearson bivariate correlations in males and females.

\begin{tabular}{|c|c|c|c|c|c|c|c|c|}
\hline \multirow[b]{2}{*}{ Parameters } & \multicolumn{4}{|c|}{ Correlation coefficients in males } & \multicolumn{4}{|c|}{ Correlation coefficients in females } \\
\hline & ALT & AST & GGT & TB & ALT & AST & GGT & TB \\
\hline Age & $-0.242^{\mathrm{a}}$ & -0.175 & -0.041 & -0.072 & -0.132 & -0.079 & $0.232^{\mathrm{a}}$ & 0.007 \\
\hline BMI & 0.017 & 0.008 & 0.047 & -0.011 & -0.005 & 0.043 & $0.355^{\mathrm{b}}$ & 0.122 \\
\hline WBC & $0.453^{\mathrm{b}}$ & $0.216^{\mathrm{a}}$ & $0.427^{\mathrm{b}}$ & 0.090 & 0.129 & 0.115 & $0.232^{\mathrm{a}}$ & -0.097 \\
\hline $\mathrm{RBC}$ & -0.129 & -0.097 & $-0.307^{\mathrm{b}}$ & 0.167 & $-0.228^{\mathrm{a}}$ & $-0.232^{\mathrm{a}}$ & -0.099 & $0.243^{\mathrm{a}}$ \\
\hline $\mathrm{Hg}$ & -0.166 & -0.152 & $-0.291^{\mathrm{b}}$ & $0.235^{\mathrm{a}}$ & -0.134 & -0.149 & -0.047 & $0.337^{\mathrm{b}}$ \\
\hline PLT & -0.086 & -0.132 & -0.032 & 0.088 & $-0.291^{\mathrm{b}}$ & $-0.306^{\mathrm{b}}$ & 0.058 & $-0.258^{a}$ \\
\hline ALT & - & $0.850^{\mathrm{b}}$ & $0.672^{\mathrm{b}}$ & 0.002 & - & $0.942^{\mathrm{b}}$ & $0.403^{\mathrm{b}}$ & 0.001 \\
\hline AST & $0.850^{\mathrm{b}}$ & - & $0.534^{\mathrm{b}}$ & -0.036 & $0.942^{\mathrm{b}}$ & - & $0.426^{\mathrm{b}}$ & -0.003 \\
\hline GGT & $0.672^{\mathrm{b}}$ & $0.534^{\mathrm{b}}$ & - & 0.080 & $0.403^{\mathrm{b}}$ & $0.426^{\mathrm{b}}$ & - & 0.161 \\
\hline TB & 0.002 & -0.036 & 0.080 & - & 0.001 & -0.003 & 0.161 & - \\
\hline BUN & $-0.237^{\mathrm{a}}$ & $-0.209^{a}$ & -0.031 & -0.048 & $-0.205^{\mathrm{a}}$ & $-0.240^{\mathrm{a}}$ & -0.021 & 0.195 \\
\hline $\mathrm{Cr}$ & -0.082 & -0.075 & 0.131 & 0.127 & -0.084 & -0.101 & 0.028 & $0.284^{b}$ \\
\hline
\end{tabular}

${ }^{\mathrm{a}} \mathrm{P}<0.05,{ }^{\mathrm{b}} \mathrm{P}<0.01$. ALT, alanine aminotransferase; AST, aspartate aminotransferase; GGT, $\gamma$-glutamyl transferase; TB, total bilirubin; BMI, body mass index; WBC, white blood cell; RBC, red blood cell; Hg, hemoglobin; PLT, platelet; BUN, blood urea nitrogen; Cr, creatinine.

Table IV. ROC indices of hepatic parameters for diagnosing or predicting IM.

A, Male

\begin{tabular}{|c|c|c|c|c|c|c|c|}
\hline Parameters & AUC & Cut-off value & Sensitivity (\%) & Specificity (\%) & Accuracy (\%) & PPV (\%) & $\operatorname{NPV}(\%)$ \\
\hline ALT & $0.764^{\mathrm{a}}$ & $27.5 \mathrm{U} / 1$ & 72.300 & 76.600 & 74.468 & 75.556 & 73.469 \\
\hline AST & $0.752^{\mathrm{a}}$ & $21.5 \mathrm{U} / 1$ & 70.200 & 72.300 & 71.277 & 71.739 & 70.833 \\
\hline GGT & $0.809^{\mathrm{a}}$ & $29.5 \mathrm{U} / 1$ & 74.500 & 76.600 & 75.532 & 76.087 & 75.000 \\
\hline TB & $0.676^{\mathrm{a}}$ & $10.70 \mu \mathrm{mol} / 1$ & 63.800 & 66.000 & 64.894 & 64.583 & 65.217 \\
\hline
\end{tabular}

B, Female

\begin{tabular}{|c|c|c|c|c|c|c|c|}
\hline Parameters & AUC & Cut-off value & Sensitivity (\%) & Specificity (\%) & Accuracy (\%) & PPV (\%) & NPV $(\%)$ \\
\hline ALT & $0.698^{\mathrm{a}}$ & $15.5 \mathrm{U} / 1$ & 60.400 & 75.000 & 67.708 & 70.732 & 65.455 \\
\hline AST & $0.742^{\mathrm{a}}$ & $17.5 \mathrm{U} / \mathrm{l}$ & 64.600 & 68.700 & 66.667 & 67.391 & 66.000 \\
\hline GGT & $0.742^{\mathrm{a}}$ & $18.0 \mathrm{U} / 1$ & 68.800 & 77.100 & 72.917 & 75.000 & 71.154 \\
\hline TB & $0.759^{a}$ & $7.45 \mu \mathrm{mol} / 1$ & 81.300 & 75.000 & 78.125 & 80.000 & 76.471 \\
\hline
\end{tabular}

${ }^{\mathrm{a}} \mathrm{P}<0.01$. ROC, receiver operating characteristic; IM, infectious mononucleosis; AUC, area under the curve; PPV, positive predictive value; NPV, negative predictive value; ALT, alanine aminotransferase; AST, aspartate aminotransferase; GGT, $\gamma$-glutamyl transferase; TB, total bilirubin.

adjusted ORs were 38.054 and 9.882, respectively (all $\mathrm{P}<0.01$ ). For TB, crude OR calculations were performed using the highest quartile as a reference. Lower TB levels were deemed to significantly increase the risk of IM in males and females. Adjusted OR calculations used age and BMI as covariates. Notably, females exhibited a higher risk of developing IM when they exhibited low serum TB. Crude ORs in quartile 1 were 8.229 and 8.257 for males and females, respectively; however, adjusted ORs were 8.883 and 10.048 , respectively (all $\mathrm{P}<0.01$ ).

\section{Discussion}

Worldwide, $>95 \%$ of adults are infected with EBV and in industrialized countries, it is estimated that $\sim 50 \%$ of the population experiences primary EBV infection $<5$ years old (1). Most people infected with EBV are either asymptomatic or exhibit mild symptoms; however, some will develop IM. Mild to moderate elevations of liver enzymes occur in $80-90 \%$ of patients with IM, however jaundice only occurs in 
A

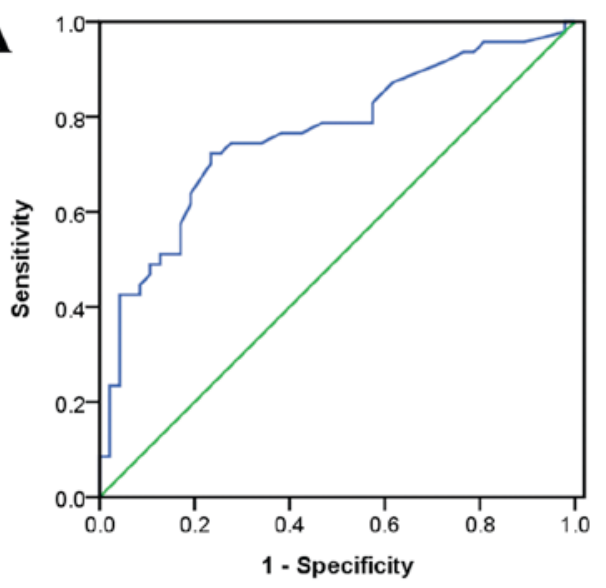

C

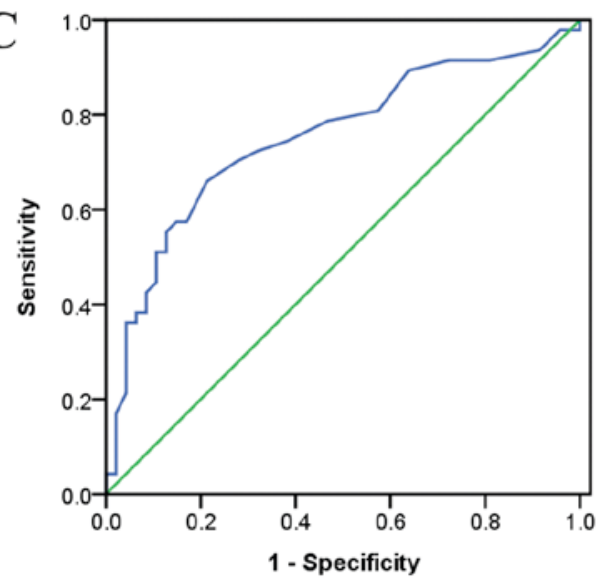

$\mathbf{E}$

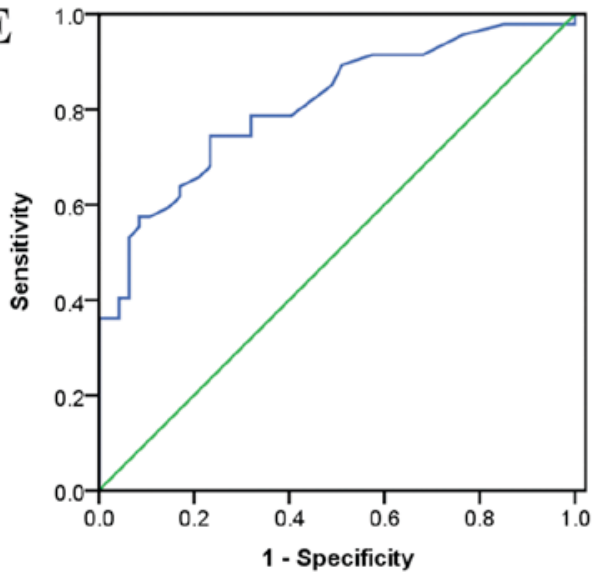

G

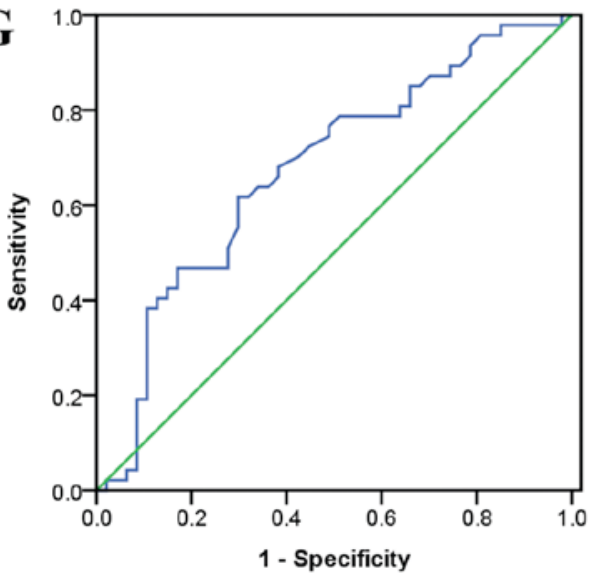

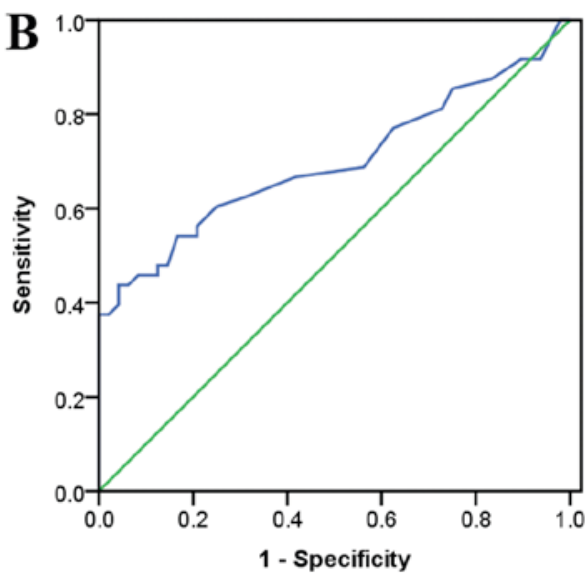

D

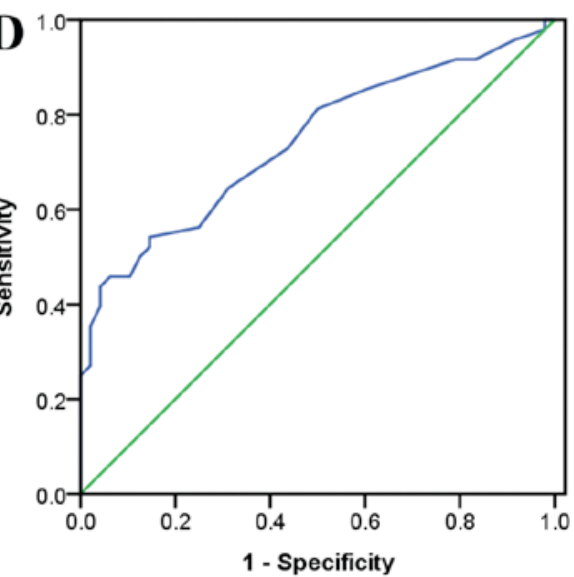

$\mathbf{F}$
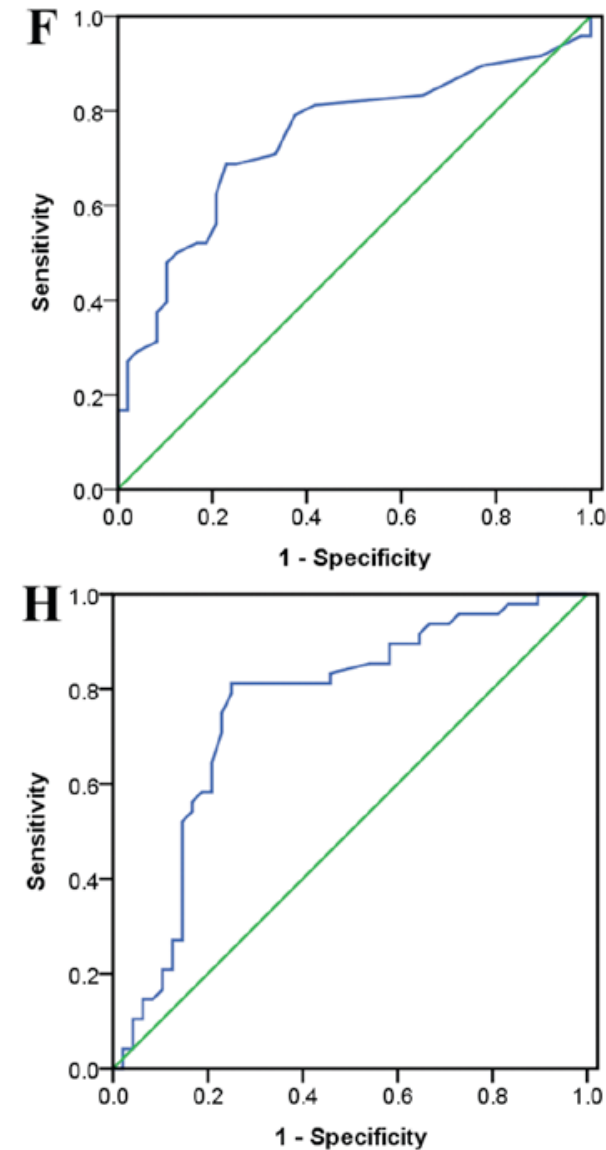

Figure 1. Diagnostic values of hepatic function indices for infectious mononucleosis in males and females analyzed by ROC curves. (A) ROC curve of alanine aminotransferase in males; (B) ROC curve of alanine aminotransferase in females; (C) ROC curve of aspartate aminotransferase in males (D) ROC curve of aspartate aminotransferase in females (E) ROC curve of $\gamma$-glutamyl transferase in males; (F) ROC curve of $\gamma$-glutamyl transferase in females; (G) ROC curve of total bilirubin in males; $(\mathrm{H})$ ROC curve of total bilirubin in females. ROC, receiver operating characteristic. 


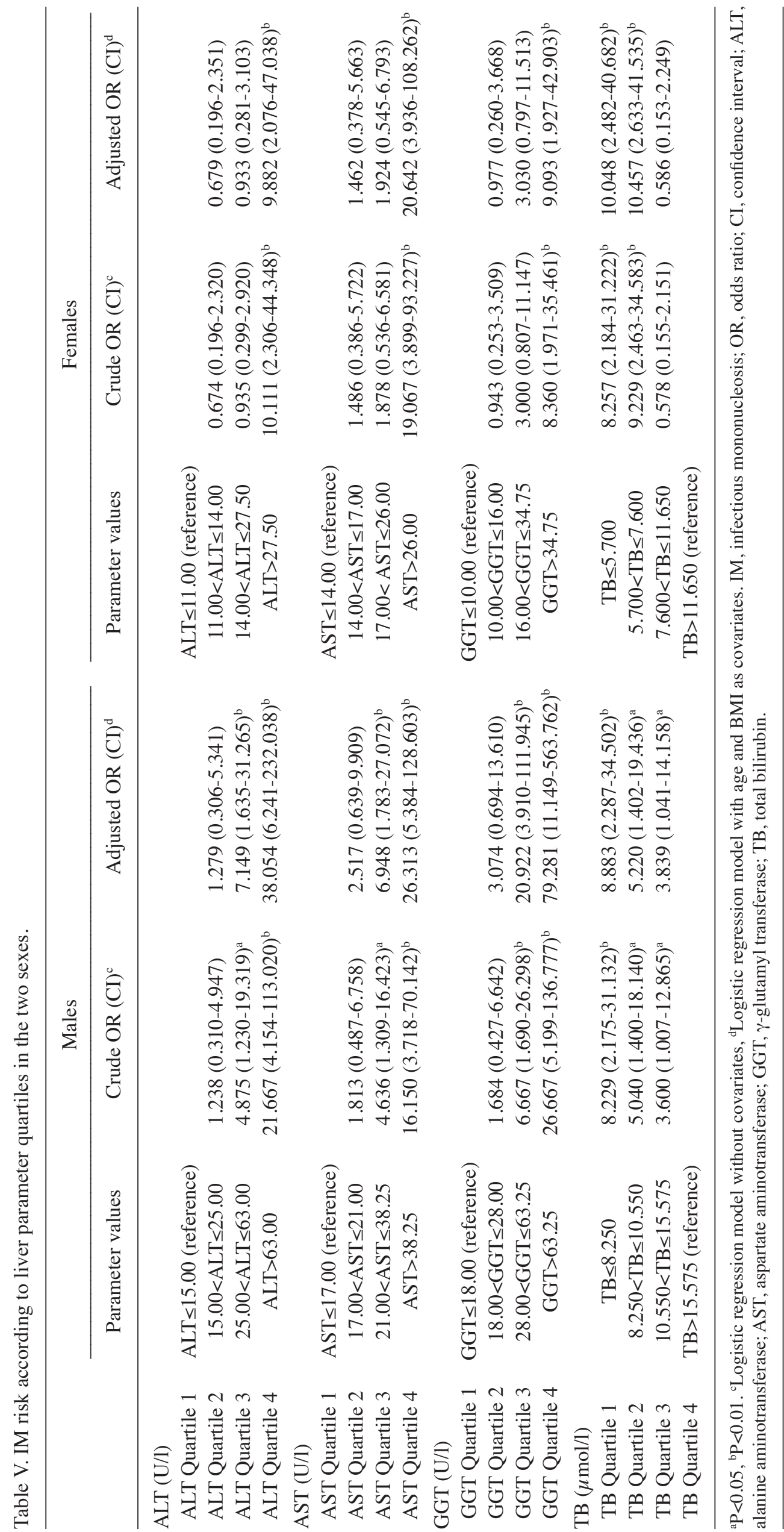


$5 \%(1-3,13)$. Furthermore, hepatic failure in patients with IM has been reported only occasionally (16-18). The three most clinically relevant transferases are ALT, AST and GGT, which are the primary indices of liver function (19). Transaminases transfer amino and keto groups between amino acids and keto acids (19). ALT catalyzes this interconversion between L-alanine and $\alpha$-ketoglutarate on one side and pyruvate and L-glutamate on the other. AST catalyzes this interconversion between aspartate and $\alpha$-ketoglutarate on one side and oxaloacetate and glutamate on the other (19). GGT belongs to the subclass of amino-acyltransferases, which transfer acyl groups between amino acids and peptides (19). GGT catalyzes the transfer from a 5-L-glutamyl-peptide and an amino acid on one side to a peptide and a 5-L-glutamyl amino acid on the other (19). Analysis of liver biopsies taken from patients with IM identified marked periportal lymphocytic infiltration, marked Kupffer cell activity and considerable acidophilic degeneration of individual liver cells (20), which may explain why transferase levels are increased in patients with IM. By contrast, patients with infectious hepatitis exhibit necrosis and inflammatory exudates (21). The results of the current study indicate that ALT, AST and GGT levels are significantly increased in males and females with IM compared with controls, indicating that transferase levels may be used to diagnose IM or determine IM risk. High transferase levels significantly increased the risk of IM, particularly in males.

Bilrubin is a by-product of heme degradation (22). The enzyme heme-oxygenase catalyzes heme and results in the formation of three products: Biliverdin, ferrous iron and carbon monoxide. Biliverdin is reduced to bilirubin by biliverdin reductase, bilirubin then binds to albumin and circulates in the blood. Bilirubin is then neutralized following its encounter with hepatocytes (22). The metabolic pathway that converts biliverdin, a soluble and nontoxic heme product, into bilirubin, which is insoluble and potentially toxic, has been evolutionarily conserved (22). It has therefore been hypothesized that bilirubin may serve an important physiological role. Previous studies have demonstrated that, although bilirubin is cytotoxic at high concentrations, at physiological concentrations bilirubin is able to scavenge free radicals and has powerful immunosuppressive effects (23-25). Indeed, the antioxidant effect of bilirubin is $\sim 20$ times more potent than that of Vitamin E, a known scavenger of free radicals (26).

Low serum bilirubin was identified as a strong, independent risk factor for coronary disease in 1994 (27) and the inverse association between bilirubin levels and coronary artery disease has been confirmed $(28,29)$. Targeting heme-oxygenase and regulating biliverdin reductase may be novel methods of treating coronary artery disease and are currently under investigation $(28,29)$. Niacin induces heme-oxygenase, which may partially explain its vascular protective properties (30). Bilirubin affects the immune system at physiological concentrations (23); for example, bilirubin inhibits the complement cascade by interrupting the binding of the $\mathrm{C} 1$ complex to the complement antibody (31). Bilirubin may also inhibit the cell-surface expression of major histocompatibility complex II molecules on antigen-presenting cells (32). Furthermore, following entry into a target cell, bilirubin may engage in the widespread inhibition of protein kinases (33). In various inflammatory and immunological disorders, including multiple sclerosis (34), Crohn's disease (35) and systemic lupus erythematosus (36), bilirubin may be depleted following completion of its functions.

One of the objectives of the current study was to investigate the association between IM and bilirubin. It has been demonstrated that oxidative stress serves a crucial role in the pathogenesis of various infections, including the hepatitis virus (37), respiratory viruses (38), human immunodeficiency virus (39), influenza virus (40), Staphylococcus aureus (41), Helicobacter pylori (42) and mycoplasma (43). It is hypothesized that an infection due to EBV may also cause oxidative stress, leading to the marked depletion of antioxidants, such as bilirubin. This may be the underlying mechanism explaining the decrease in bilirubin observed in patients with IM in the current study. Furthermore, the present study indicates that females with low bilirubin levels are more susceptible to developing IM than males with low bilirubin levels. Males generally exhibit much higher serum bilirubin than females (44) and this was confirmed in the current study. Higher bilirubin levels may confer more antioxidant protection in males, therefore females may be more susceptible to the oxidative stress caused by bilirubin depletion. Consequently, decreased bilirubin may be more predictive of IM infection in females than males.

In the present study, there were differences between males and females among indices other than liver function parameters. Cr and BUN levels, as well as WBC levels, were significantly higher in males than in females. Previous studies have demonstrated that $\mathrm{Cr}$ levels are equal in children; however during adolescence, $\mathrm{Cr}$ levels increase more in males than females $(45,46)$. In adulthood, females have lower serum $\mathrm{Cr}$ values than males with similar renal function (47). $\mathrm{Cr}$ and BUN induce the same effects in males and females (48-53). The results of studies investigating WBC levels are inconsistent; certain studies indicate that WBC levels are higher in males $(54,55)$, whereas others indicate that WBC levels are higher in females $(56,57)$. However, major influential factors include age and sex hormone levels; indeed, it has been suggested that WBC levels may change in females at different stages of the menstrual cycle (54). In addition, liver function parameters are associated with age, body fat, renal function and blood cells (48-53) and correlations between these key variables were identified in the current study.

There were several limitations of the current study. Firstly, the cross-sectional design of the current investigation meant that no causality could be determined. A prospective study should therefore be performed in the future. Secondly, a limited number of patients with IM and controls were evaluated and they were only recruited from a single center. More participants should be recruited from multiple centers in order to verify the results of the current study. Thirdly, due to budget limitations of the current study, measurements of serum parameters were based on a single determination and markers of inflammation or activities of antioxidants were not assessed. Finally, the administration of bilirubin as a possible adjuvant therapy should be investigated in the future to validate the results of the current study.

In conclusion, the results of the current study identified a positive association between transferases and IM and a negative association between TB and IM, suggesting that there is a progressive decrease of antioxidant reserves during IM. High 
transferase levels were suggestive of IM, particularly in males, whereas low TB was suggestive of IM, particularly in females.

\section{Acknowledgements}

The present study was supported by the National Key Clinical Specialty Project; the Tianjin Medical University General Hospital New Century Excellent Talent Program; the Young and Middle-aged Innovative Talent Training Program from Tianjin Education Committee; and the Talent Fostering Program (the 131 Project) from Tianjin Education Committee, Tianjin Human Resources and Social Security Bureau. The present study was also supported by China National Natural Science Foundation (grant no. 81571709), the Key Project of Tianjin Science and Technology Committee Foundation (grant no. 16JCZDJC34300); and the Tianjin Science and Technology Committee Foundation (grant nos. 11ZCGYSY05700, 12ZCZDSY20400 and 13ZCZDSY20200).

\section{References}

1. Luzuriaga K and Sullivan JL: Infectious mononucleosis. N Engl J Med 362: 1993-2000, 2010.

2. Vouloumanou EK, Rafailidis PI and Falagas ME: Current diagnosis and management of infectious mononucleosis. Curr Opin Hematol 19: 14-20, 2012

3. Wang Y, Li J, Ren YY and Zhao H: The levels of liver enzymes and atypical lymphocytes are higher in youth patients with infectious mononucleosis than in preschool children. Clin Mol Hepatol 19: 382-388, 2013.

4. Topp SK, Rosenfeldt V, Vestergaard H, Christiansen CB and Von Linstow ML: Clinical characteristics and laboratory findings in Danish children hospitalized with primary Epstein-Barr virus infection. Infect Dis (Lond) 47: 908-914, 2015.

5. Cohn C and Lidman BI: Hepatitis without jaundice in infectious mononucleosis. J Clin Invest 25: 145-151, 1946.

6. Rosalki SB, Jones TG and Verney AF: Transaminase and liver-function studies in infectious mononucleosis. Br Med J 1: 929-932, 1960.

7. Horwitz CA, Burke MD, Grimes P and Tombers J: Hepatic function in mononucleosis induced by Epstein-Barr virus and cytomegalovirus. Clin Chem 26: 243-246, 1980.

8. Yang SI, Geong JH and Kim JY: Clinical characteristics of primary Epstein Barr virus hepatitis with elevation of alkaline phosphatase and $\gamma$-glutamyltransferase in children. Yonsei Med J 55: 107-112, 2014

9. Uluğ M, Celen MK, Ayaz C, Geyik MF and Hoşoğlu S: Acute hepatitis: A rare complication of Epstein-Barr virus (EBV) infection. J Infect Dev Ctries 4: 668-673, 2010.

10. Zenda T, Itoh Y, Takayama Y, Masunaga T, Asaka S, Oiwake H, Shinozaki K and Takeda R: Significant liver injury with dual positive IgM antibody to Epstein-Barr virus and cytomegalovirus as a puzzling initial manifestation of infectious mononucleosis. Intern Med 43: 340-343, 2004.

11. Baron DN, Bell JL and Dunnet WN: Biochemical studies on hepatic involvement in infectious mononucleosis. J Clin Pathol 18: 209-211, 1965.

12. Evans AS: Liver involvement in infectious mononucleosis. J Clin Invest 27: 106-110, 1948.

13. Straus SE, Cohen JI, Tosato G and Meier J: NIH conference. Epstein-Barr virus infections: Biology, pathogenesis, and management. Ann Intern Med 118: 45-58, 1993.

14. Hu L, Yang J, Cui T, Xing H and Cai P: Diagnosis of infectious mononucleosis by combined detection of atypical lymphocytes and transaminase. J Huazhong Univ Sci Technolog Med Sci 26: 384-385, 2006.

15. DeLong ER, DeLong DM and Clarke-Pearson DL: Comparing the areas under two or more correlated receiver operating characteristic curves: A nonparametric approach. Biometrics 44: 837-845, 1988.

16. Ainley NJ: A fatal case of infectious mononucleosis with extensive zonal necrosis of the liver. Ulster Med J 18: 219-224, 1949.
17. Harries JT and Ferguson AW: Fatal infectious mononucleosis with liver failure in two sisters. Arch Dis Child 43: 480-485, 1968.

18. McMahon JM, Elliott CW and Green RC: Infectious mononucleosis complicated by hepatic coma. Am J Gastroenterol 51: 200-207, 1969.

19. Herbinger KH, Hanus I, Felbinger TW, Weber C, Beissner M, von Sonnenburg F, Löscher T, Bretzel G, Nothdurft HD, Hoelscher M and Alberer M: Elevated values of clinically relevant transferases induced by imported infectious diseases: A controlled cross-sectional study of 14,559 diseased german travelers returning from the tropics and subtropics. Am J Trop Med Hyg 95: 481-487, 2016.

20. Wadsworth RC and Keil PG: Biopsy of the liver in infectious mononucleosis. Am J Pathol 28: 1003-1025, 1952.

21. Sullivan BH Jr, Irey NS, Pileggi VJ, Crone RI and Gibson JR: The liver in infectious mononucleosis. Am J Dig Dis 2: 210-223, 1957.

22. Maines MD: The heme oxygenase system: A regulator of second messenger gases. Annu Rev Pharmacol Toxicol 37: 517-554, 1997.

23. Jangi S, Otterbein L and Robson S: The molecular basis for the immunomodulatory activities of unconjugated bilirubin. Int J Biochem Cell Biol 45: 2843-2851, 2013.

24. Kapitulnik J: Bilirubin: An endogenous product of heme degradation with both cytotoxic and cytoprotective properties. Mol Pharmacol 66: 773-779, 2004.

25. Stocker R, Yamamoto Y, McDonagh AF, Glazer AN and Ames BN: Bilirubin is an antioxidant of possible physiological importance. Science 235: 1043-1046, 1987.

26. Wu TW, Fung KP and Yang CC: Unconjugated bilirubin inhibits the oxidation of human low density lipoprotein better than Trolox. Life Sci 54: P477-P481, 1994.

27. Schwertner HA, Jackson WG and Tolan G: Association of low serum concentration of bilirubin with increased risk of coronary artery disease. Clin Chem 40: 18-23, 1994.

28. Akboga MK, Canpolat U, Sahinarslan A, Alsancak Y, Nurkoc S, Aras D, Aydogdu S and Abaci A: Association of serum total bilirubin level with severity of coronary atherosclerosis is linked to systemic inflammation. Atherosclerosis 240: 110-114, 2015.

29. Ghem C, Sarmento-Leite RE, de Quadros AS, Rossetto S and Gottschall CA: Serum bilirubin concentration in patients with an established coronary artery disease. Int Heart J 51: 86-91, 2010.

30. Wu BJ, Chen K, Barter PJ and Rye KA: Niacin inhibits vascular inflammation via the induction of heme oxygenase-1. Circulation 125: 150-158, 2012.

31. Basiglio CL, Arriaga SM, Pelusa HF, Almará AM, Roma MG and Mottino AD: Protective role of unconjugated bilirubin on complement-mediated hepatocytolysis. Biochim Biophys Acta 1770: 1003-1010, 2007.

32. Wu J, Ma J, Fan ST, Schlitt HJ and Tsui TY: Bilirubin derived from heme degradation suppresses MHC class II expression in endothelial cells. Biochem Biophys Res Commun 338: 890-896, 2005.

33. Hansen TW, Mathiesen SB and Walaas SI: Bilirubin has widespread inhibitory effects on protein phosphorylation. Pediatr Res 39: 1072-1077, 1996.

34. Peng F, Deng X, Yu Y, Chen X, Shen L, Zhong X, Qiu W, Jiang Y, Zhang $\mathbf{J}$ and $\mathrm{Hu} \mathrm{X}$ : Serum bilirubin concentrations and multiple sclerosis. J Clin Neurosci 18: 1355-1359, 2011.

35. Liechti FD, Grandgirard D and Leib SL: Bacterial meningitis: Insights into pathogenesis and evaluation of new treatment options: A perspective from experimental studies. Future Microbiol 10: 1195-1213, 2015.

36. Vitek L, Muchová L, Jančová E, Pešičková S, Tegzová D, Peterová V, Pavelka K, Tesař V and Schwertner H: Association of systemic lupus erythematosus with low serum bilirubin levels. Scand J Rheumatol 39: 480-484, 2010.

37. Zuwała-Jagiełło J, Warwas $M$ and Pazgan-Simon $M$ : Ischemia-modified albumin (IMA) is increased in patients with chronic hepatitis $\mathrm{C}$ infection and related to markers of oxidative stress and inflammation. Acta Biochim Pol 59: 661-667, 2012.

38. Komaravelli $\mathrm{N}$ and Casola A: Respiratory viral infections and subversion of cellular antioxidant defenses. J Pharmacogenomics Pharmacoproteomics 5: pii: 1000141, 2014.

39. Ngondi JL, Oben J, Forkah DM, Etame LH and Mbanya D: The effect of different combination therapies on oxidative stress markers in HIV infected patients in Cameroon. AIDS Res Ther 3: 19, 2006. 
40. Checconi P, Salzano S, Bowler L, Mullen L, Mengozzi M, Hanschmann EM, Lillig CH, Sgarbanti R, Panella S, Nencioni L, et al: Redox proteomics of the inflammatory secretome identifies a common set of redoxins and other glutathionylated proteins released in inflammation, influenza virus infection and oxidative stress. PLoS One 10: e0127086, 2015.

41. Chakraborty SP, Das S, Chattopadhyay S, Tripathy S, Dash SK Pramanik P and Roy S: Staphylococcus aureus infection induced redox signaling and DNA fragmentation in T-lymphocytes: Possible ameliorative role of nanoconjugated vancomycin. Toxicol Mech Methods 22: 193-204, 2012.

42. Aslan M, Nazligul Y, Horoz M, Bolukbas C, Bolukbas FF, Aksoy N, Celik $\mathrm{H}$ and Erel O: Serum prolidase activity and oxidative status in Helicobacter pylori infection. Clin Biochem 40: 37-40, 2007.

43. Kariya C, Chu HW, Huang J, Leitner H, Martin RJ and Day BJ: Mycoplasma pneumoniae infection and environmental tobacco smoke inhibit lung glutathione adaptive responses and increase oxidative stress. Infect Immun 76: 4455-4462, 2008.

44. Rosenthal P, Pincus M and Fink D: Sex- and age-related differences in bilirubin concentrations in serum. Clin Chem 30 1380-1382, 1984.

45. Pottel H, Vrydags N, Mahieu B, Vandewynckele E, Croes K and Martens F: Establishing age/sex related serum creatinine reference intervals from hospital laboratory data based on different statistical methods. Clin Chim Acta 396: 49-55, 2008.

46. Uemura O, Honda M, Matsuyama T, Ishikura $\mathrm{K}$, Hataya $\mathrm{H}$, Yata N, Nagai T, Ikezumi Y, Fujita N, Ito S, et al: Age, gender, and body length effects on reference serum creatinine levels determined by an enzymatic method in Japanese children: A multicenter study. Clin Exp Nephrol 15: 694-699, 2011.

47. O'Leary JG, Wong F, Reddy KR, Garcia-Tsao G, Kamath PS, Biggins SW, Fallon MB, Subramanian RM, Maliakkal B, Thacker L and Bajaj JS: Gender-specific differences in baseline, peak, and delta serum creatinine: The NACSELD experience. Dig Dis Sci 62: 768-776, 2017.

48. Meng Z, Liu M, Zhang Q, Liu L, Song K, Tan J, Jia Q, Zhang G, Wang R, He Y, et al: Gender and age impacts on the association between thyroid function and metabolic syndrome in Chinese. Medicine (Baltimore) 94: e2193, 2015

49. Meng Z, Liu M, Zhang Q, Liu L, Song K, Tan J, Jia Q, Zhang G, Wang R, He Y, et al: Gender and age impact on the association between thyroid-stimulating hormone and serum lipids. Medicine (Baltimore) 94: e2186, 2015.
50. Ren X, Meng Z, Liu M, Zhu M, He Q, Zhang Q, Liu L, Song K, Jia Q, Jia Q, et al: No associations exist between mean platelet volume or platelet distribution width and thyroid function in Chinese. Medicine (Baltimore) 95: e4573, 2016.

51. Zhou P, Meng Z, Liu M, Ren X, Zhu M, He Q, Zhang Q, Liu L, Song K, Jia Q, et al: The associations between leukocyte, erythrocyte or platelet, and metabolic syndrome in different genders of Chinese. Medicine (Baltimore) 95: e5189, 2016.

52. Wang S, Zhang J, Zhu L, Song L, Meng Z, Jia Q, Li X, Liu N, $\mathrm{Hu}$ T, Zhou P, et al: Association between liver function and metabolic syndrome in Chinese men and women. Sci Rep 7: 44844, 2017.

53. Zhang J, Meng Z, Zhang Q, Liu L, Song K, Tan J, Li X, Jia Q, Zhang $\mathrm{G}$ and $\mathrm{He} \mathrm{Y}$ : Gender impact on the correlations between subclinical thyroid dysfunction and hyperuricemia in Chinese. Clin Rheumatol 35: 143-149, 2016.

54. Nowak J, Borkowska B and Pawlowski B: Leukocyte changes across menstruation, ovulation, and mid-luteal phase and association with sex hormone variation. Am J Hum Biol 28: 721-728, 2016.

55. Pérez-de-Heredia F, Gómez-Martínez S, Díaz LE, Veses AM, Nova E, Wärnberg J, Huybrechts I, Vyncke K, Androutsos O, Ferrari M, et al: Influence of sex, age, pubertal maturation and body mass index on circulating white blood cell counts in healthy European adolescents-the HELENA study. Eur J Pediatr 174: 999-1014, 2015.

56. Mandala WL, Gondwe EN, MacLennan JM, Molyneux ME and MacLennan CA: Age- and sex-related changes in hematological parameters in healthy Malawians. J Blood Med 8: 123-130, 2017.

57. Pratley RE, Wilson C and Bogardus C: Relation of the white blood cell count to obesity and insulin resistance: Effect of race and gender. Obes Res 3: 563-571, 1995.

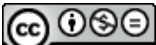

This work is licensed under a Creative Commons Attribution-NonCommercial-NoDerivatives 4.0 International (CC BY-NC-ND 4.0) License. 